\title{
A comparison of resistance to satiation and resistance to extinction
}

\author{
ELIZABETH D. CAPALDI, DAVID E. MYERS, and T. L. DAVIDSON \\ Purdue University, West Lafayette, Indiana 47907
}

\begin{abstract}
In two experiments, resistance to satiation was compared with resistance to extinction. In Experiment 1, rats given initial trials in a straight-alley runway while satiated failed to show increased resistance to satiation in a later test phase. This negative finding contrasts with the increased resistance to extinction usually found following initial nonrewarded trials in a straight alley. In Experiment 2, rats were extinguished or were run while satiated following deprived acquisition, and then were either shifted to the other condition or maintained under the same condition. A greater response decrement was produced by extinction than by satiation, both when current performance was examined and when the persistent effect of satiation or extinction on later performance was examined. These results show that there are important dissimilarities in the effects of satiation and extinction, dissimilarities that suggest that extinction is more nonrewarding or aversive than satiation. It seems likely that extinction involves processes (such as frustration, arousal of aversive motivation, and conditioned inhibition) not involved in satiation, which account for the greater response decrement in extinction as compared with satiation.
\end{abstract}

Behavior often continues after its original motivation is no longer present. In everyday life, responses learned early in life under one set of motivationreward conditions continue to occur later in life when the original motivation is no longer present. A robust example of this phenomenon in the laboratory is the continued performance by satiated rats of instrumental responses learned previously under hunger, a phenomenon labeled "resistance to satiation" by Morgan (1974). Rats continue to run an alley for food and to eat the food when tested while satiated after having been trained hungry (e.g., Capaldi \& Myers, 1978). This phenomenon of continued running and eating is notable because satiated rats will not learn to run an alley if they are initially trained while satiated (e.g., Cox, 1976; Seward, Shea, \& Elkind, 1958). Eating and running for food under satiation are a result of previous training under hunger.

Many explanations of resistance to satiation have been offered. In 1952, Webb reviewed these explanations, dividing then into three types: nominal theories, irrelevant drive theories, and acquired tendency theories-the latter including acquired drive theories and stimulus evocation theories. Nominal theories are those that name the phenomenon, for example, "functional autonomy" (Allport, 1937). Few predic-

Experiment 2 is based on a thesis by the second author submitted in December 1977 to the Graduate School of Purdue University in partial fulfillment of the requirements for the Master of Science degree. Appreciation is expressed to Hali R. Myers for construction of the figures. tions stem from these theories, and they have stimulated little research. Irrelevant drive theories (e.g., Hull, 1943) are those suggesting that any drive can energize any habit. There is little evidence to support the idea that any drive can energize any habit (see Bolles, 1975, for a review), so it seems unlikely that resistance to satiation is a result of irrelevant drives energizing the habit formed in acquisition. Data collected since 1952 have also rendered explanation of resistance to satiation in terms of acquired drive less feasible than it seemed in 1952 (see Cravens \& Renner, 1970). Capaldi and Myers' (1978) findings also suggest that resistance to satiation cannot be totally attributed to irrelevant drive or conditioned drive. These findings suggest, too, that incomplete satiation cannot account for resistance to satiation. In these studies, schedule of reward and magnitude of reward received in acquisition were shown to influence resistance to satiation. Because all groups in Capaldi and Myers' studies initially received the same number of trials under the same level of food deprivation, and then were tested following the same satiation procedure, the groups should have been equal in terms of any possible conditioned hunger, in degree of satiation at the time of testing, and in irrelevant drive. Yet, the groups differed greatly in resistance to satiation.

Of the theories reviewed by Webb, stimulus evocation theories now seem to be the most feasible. Stimulus evocation theories suggest that behavior that was originally drive-produced or drive-motivated may occur under satiation purely on associative grounds. As stated by Capaldi and Myers (1978), the original stimulus situation will elicit the responses 
conditioned to it by "force of habit." Capaldi and Myers found that rats were more resistant to satiation following partial reward training than they were following consistent reward training, and following small reward training than following large reward training, whether a partial or consistent reward schedule was used. These data can be interpreted as indicating that the instrumental response and eating occur in satiation because the cues present in satiated testing, including both external cues and internal cues produced by the memory of reward, elicit responses associated with them in acquisition. Resistance to satiation is greater following small reward or partial training because, during testing while satiated, rats eat only a small reward (even if a large reward is given in satiated testing, rats eat only a small part of the reward). The memory of reward from Trial $n-1$ that is present on Trial $n$ of satiated testing is characteristic of a small reward. This is the memory associated with responding for rats trained with a small reward, and is similar to the memory of nonreward associated with responding for rats trained with partial reward (see Capaldi \& Myers, 1978, for a detailed discussion of this view).

An alternative (but not incompatible) stimulus evocation explanation of resistance to satiation is that expectancies of reward continue to produce responding in satiation. As stated by Morgan (1974), "If lever pressing has been reinforced, this may be sufficient reason for it to occur again. The rat will have to find out by experience that in the changed state resulting from a change in deprivation condition, lever pressing is no longer reinforced" (p. 458). Morgan also suggested that resistance to satiation might therefore be viewed as similar to resistance to extinction, because in both cases the animal must learn that reward is no longer forthcoming (extinction of the expectancy of reward or of incentive motivation).

Within Capaldi and Myers' (1978) interpretation, resistance to extinction and resistance to satiation are also seen as similar, because in both cases responding will be affected by the similarity of internal and external stimuli present in training and test. However, Capaldi and Myers suggested that extinction and satiation may also be different in important ways. For example, perhaps the expectancy or receiving a high-value reward does not transfer from high deprivation to satiation (see Capaldi \& Myers, 1978, for supporting evidence). In contrast, expectancies of the reward value received in acquisition do seem to transfer from acquisition to extinction. And receiving nonreward in the presence of an expectancy of receiving reward has been shown to produce an emotional state labeled frustration (Amsel, 1958; Daly, 1974). If expectancies of receiving a highvalue reward do not transfer from high deprivation to satiation, satiated-food testing will not be frustrating, and satiation thus differs from extinction in being nonfrustrative.

There is no clear evidence on whether satiated testing is frustrating. Actually, there is no clear evidence that satiated testing is even nonrewarding. In satiated testing, food is still received, and, while it seems reasonable to assume that the value of food is reduced by satiation, it may not be eliminated. Because of the lack of data, and because some have suggested that satiated-food testing may indeed be frustrating (Mandler, 1964; Morgan, 1974; Morris, Einon, \& Morgan, 1976), the present experiments were designed to compare extinction and satiation directly to determine the extent to which satiation may be less aversive or nonrewarding than extinction.

\section{EXPERIMENT 1}

In Experiment 1, a first step was made toward evaluating the extent to which satiated-food testing following deprived acquisition is nonrewarding. It is fairly clear that initial satiated-food testing (i.e., with no prior deprived experience) is not rewarding. Many studies have measured initial acquisition to food under satiation (e.g., Cox, 1976; Seward, Shea, \& Elkind, 1958) and have obtained no increase in performance, indicating no reinforcing or rewarding effect. Accordingly, if satiated-food testing after deprived acquisition is also not rewarding, rats given prior experience with satiated-food training should be more resistant to satiation than rats not given prior experience with satiated-food training.

It might be agreed that, while initial satiated-food training is not reinforcing, it is also not nonrewarding or aversive. In contrast, satiated-food testing after deprived acquisition may be nonrewarding or aversive. But, if so, on the basis of analogous experiments involving nonrewarded trials, there should still be positive transfer from initial satiated training to satiated test trials, if both are nonrewarding. Initial nonrewarded trials have been shown to increase resistance to extinction (e.g., Spear \& Spitzner, 1967), an effect referred to as the initial nonreward effect (INE). The INE is a robust phenomenon, relatively insensitive to procedural variations. It occurs with water deprivation and water reward (Robbins, Chait, \& Weinstock, 1968), with a large or small food reward (Capaldi \& Haggbloom, 1974), and with a long or short intertrial (Spear \& Spitzner, 1967). It has been reported in seven different experiments (Capaldi \& Haggbloom, 1974; Robbins et al., 1968; Spear, Hill, \& O'Sullivan, 1965; Spear \& Spitzner, 1967). The INE has been attributed to prior experience with nonreward in the INE group, reducing the stimulus generalization decrement associated with receiving nonreward in the test phase (Spear \& Spitzner, 1967). 
This occurs even though rats in the INE group should not be frustrated on their initial nonrewarded trials, yet presumably are frustrated in the test phase. No frustration should occur on the initial nonreward trials because an expectation of receiving reward is a necessary condition for frustration, and there is no reason for an expectation of reward to be present on the initial nonreward trials.

Experiment 1 was designed to determine if the running of rats initially satiated in a straight-alley runway, prior to high-deprivation training, increases subsequent resistance to satiation. If initial training under satiation increases subsequent resistance to satiation, it would bolster the argument that satiatedfood testing is nonrewarding and analogous to extinction.

\section{Method}

Subjects. The subjects were 30 male 90 -day-old rats purchased from the Holtzman Company, Madison, Wisconsin.

Apparatus. The apparatus was a runway painted a flat gray throughout, $208.1 \times 10.2 \mathrm{~cm}$, with $22.0-\mathrm{cm}$-high sides covered with 1.3-cm hardware cloth. A 20.3-cm-long floor treadle, suspended over a microswitch, constituted the initial portion of the alley, and when the treadle was depressed by the weight of the rat, a .01 -sec clock was started. The clock was stopped (to define start time) and another was started when the rat interrupted a photobeam $5.08 \mathrm{~cm}$ beyond the treadle. The second clock was stopped (run time) and a third one was started when the rat broke the second photobeam located $132.1 \mathrm{~cm}$ from the first. Interruption of the third photobeam, $38.8 \mathrm{~cm}$ beyond the second, stopped the final clock (goal time). The third photobeam was located $8.5 \mathrm{~cm}$ in front of a teaspoon that was attached to a block of wood and served as a food cup. A manually lowered guillotine door, $27.8 \mathrm{~cm}$ from the rear of the goal compartment, served to confine the rat to the goal area. The times from the three clocks were summed to obtain total time.

Procedure. The rats were given ad-lib food (Wayne Lab Blox) and water for 9 days following arrival in the laboratory and were assigned to three groups matched on the mean ad-lib body weight over the last 3 days. Over the next 26 days, each rat was handled for $30 \mathrm{sec}$ a day, and two of the groups (Groups D and N) were fed amounts of Wayne Lab Blox to reduce their body weights to $80 \%( \pm 1 \%)$ of their ad-lib weights, while the third group (Group S) was maintained on ad-lib food. On the next 2 days, Groups $S$ and $D$ received one trial per day in the alley and were rewarded with two $.045-\mathrm{g}$ Noyes pellets, while Group $\mathbf{N}$ was not run but was picked up when their turn in sequence arrived. Throughout the experiment, rats were run randomly within squads of six, composed of two rats from each group, producing an intertrial interval of 4-8 min. On each trial, a rat was allowed $60 \mathrm{sec}$ to leave an alley section before it was gently guided to the next section, and $60 \mathrm{sec}$ was recorded for the untraversed section. Phase 1 began the next day and lasted for 18 days, during which Groups D and $S$ received three trials per day and Group $\mathbf{N}$ was picked up in turn. Three schedules of rewarded $(R)$ and nonrewarded $(N)$ trials were employed six times in the following order: RNR, NNR, and NRR. On $\mathbf{N}$ trials, rats were removed from the goalbox after $20 \mathrm{sec}$. On R trials, rats in Group D were removed from the goalbox immediately after consuming the reward, while rats in Group $S$ were removed after consuming the reward or after $20 \mathrm{sec}$, whichever came first. Goalbox eating was recorded on every trial for Group S. Following Phase 1, rats in Group S were reduced over a 25 -day period to $80 \%( \pm 1 \%)$ of their ad-lib weights, while Groups $D$ and $N$ were maintained at $80 \%( \pm 1 \%)$ of their ad-lib weights. Over the next 2 days, Group $N$ received preliminary training in a manner identical to that given Groups $D$ and $S$ before
Phase 1. Eighteen days of Phase 2 followed, during which all three groups were run according to a procedure identical to that for Group D in Phase 1. After the last day of Phase 2, all groups were given ad-lib food, and Phase 3 began 16 days later, when all rats had stabilized above their ad-lib weights. Ten minutes before running on each day of Phase 3, a dish of 50 .045-g Noyes pellets was placed in the cage of each rat and removed following its daily trials. No attempt was made to satiate rats with Noyes pellets prior to running. Capaldi and Myers (1979) explicitly investigated the effects of satiating rats with Noyes pellets in the home cages prior to satiated testing and found that, although resistance to satiation was less for rats satiated with Noyes pellets than for those satiated with Wayne Lab Blox alone, rats satiated with Noyes pellets were highly resistant to satiation in both eating and running. Phase 3 lasted for 12 days, during which rats received three rewarded trials per day. All other procedures were identical to that of Group S in Phase 1.

\section{Results}

The data from each phase were analyzed separately in between-within analyses of variance, in which trials and days were within factors and groups was a between factor. Since the effect of trials was the same for all groups, it will not be mentioned further. Results were substantially the same in all alley sections; thus, only mean $\log$ total times $\left[\log _{10}(\sec +1)\right]$ are reported. Figure 1 presents the mean log total times for Groups D and S (Group N was not run) in Phase 1 and for all groups in Phases 2 and 3.

Phase 1. As can be seen in Figure 1, Group D ran significantly faster than Group $S$ in Phase $1[\mathrm{~F}(1,18)=$ $306.84, p<.001]$. Only two subjects in Group $S$ ate any pellets in the alley (one ate on 10 trials, and one ate on 1 trial), while, of course, each rat in Group D ate all of the pellets on every rewarded trial.

Phase 2. Figure 1 shows that, during Phase 2, Group D maintained stable, fast running times, while Groups $\mathbf{N}$ and $\mathbf{S}$ were comparable in their rate of approach to the level of performance in Group D. Analysis of variance for the three groups supported these impressions. There was a significant main effect of groups $[\mathrm{F}(2,27)=6.98, \mathrm{p}<.01]$, as well as a Groups by Days interaction $[F(34,459)=13.71$, $p<.001]$. Newman-Keuls tests on the differences between the group mean running times on each day showed that Group D was significantly faster (ps $<$ .05) than Group S on Days 1-7 and than Group N on Days 1-6, and not significantly different from either group thereafter. Groups $\mathbf{N}$ and $\mathbf{S}$ did not differ on any day in Phase 2.

Phase 3. As can be seen in Figure 1, all three groups were slower in Phase 3 than they had been under high deprivation in Phase 2, as would be expected from the decrease in hunger. More important, in Phase 3, groups were considerably faster than Group $S$ had been in Phase 1-this demonstrates the basic resistance to satiation phenomenon. However, the groups did not differ in resistance to satiation in Phase 3. During Phase 3 , all groups increased running times over days $[F(12,324)=3.52, p<.001]$, but there were no differences between groups $[F<1$; Groups by 


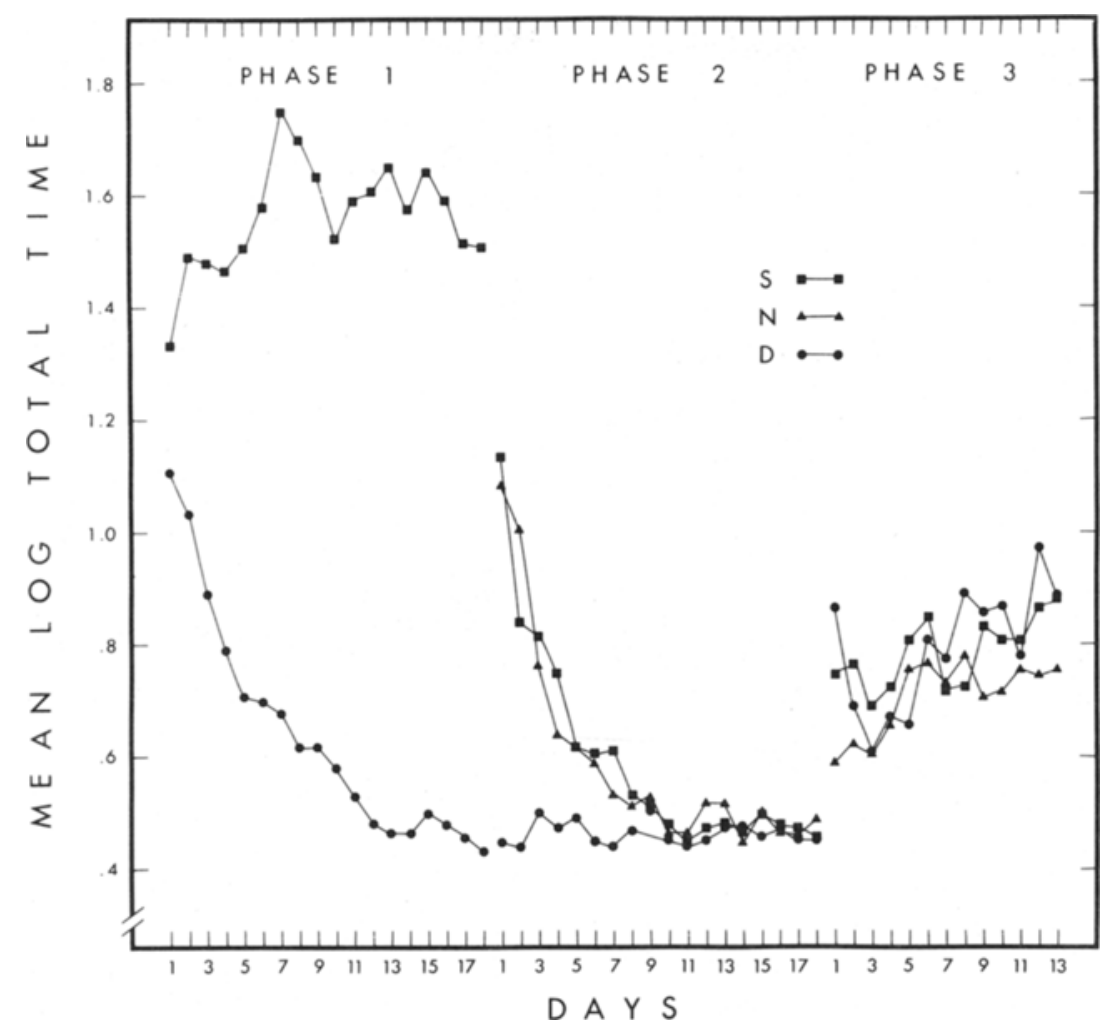

Figure 1. Mean log total times for each group, on each day, in each phase of the experiment. In Phase 1, Group N was not run, Group S was run while satiated, and Group D was run while deprived. In Phase 2, all groups were run while deprived, and in Phase 3, all groups were run while satialed.

Days, $F(24,324)=1.06, p>.38$. During Phase 3, the mean numbers of pellets eaten each day of the 50 pellets placed in the home cage by Groups S, N, and $\mathrm{D}$ were 49.8 and 49.0 , respectively. The mean numbers of trials on which eating occurred in the alley for each group were: Group S, 2.88; Group N, 2.98; and Group D, 2.88. These group differences were not significant $[F(2,27)=1.80, p>.18$, and $F<1$, respectively].

\section{Discussion}

There was no effect of initial satiated trials on performance in Phases 2 and 3. Group S did not differ from Group $N$ (which received no Phase 1 training) in Phases 2 and 3. Thus, under the conditions used in Experiment 1, there was no "initial satiated effect" analogous to the INE. As indicated above, one interpretation of the INE is that the stimulus generalization decrement associated with receiving nonreward in Phase 3 is reduced for rats that have previously received nonreward in Phase 1. Applying a similar analysis to Experiment 1, it would be expected that less of a generalization decrement would occur for Group $S$ when shifted to satiation than would occur for Groups N and D, which never before experienced satiated running trials. It could be objected that the cues associated with being satiated were not conditioned to the response in Phase 1 , inasmuch as no acquisition curve and little eating occurred in Phase 1. But the same argument can be applied to studies of the INE-no acquisition curve or eating occurs, yet initial nonrewarded trials increase subsequent resistance to extinction. Of course, the failure to find any effect of giving initial satiated trials on later resistance to satiation could be related to the specific procedures used in Experiment 1. But, because the INE is relatively insensitive to procedural variations, as indicated above, it is interesting to speculate on what theoretical reasons there may be for the lack of group differences in Experiment 1.

One interpretation of the results of Experiment 1 is that satiated testing after deprivation is very different from satiated testing prior to deprivation training. Thus, the stimulus generalization decrement resulting from shifting to satiated testing in Phase 3 is present for Group $S$, as it is for groups that were never run while satiated prior to Phase 3 . Some as- 
pects of the data, aside from the lack of differences between groups in Phase 3, suggest that satiated alley exposure prior to deprivation training is different from satiated testing after deprivation training. In Phase 1 , only one subject in Group $S$ consistently ate the pellets, but, in Phase 3, all groups ate the pellets on almost every rewarded trial. Also, performance during Phase 3 for all groups was dramatically better than the performance of Group $S$ in Phase 1. Although animals did show increased running times over days in Phase 3 , suggesting that Phase 3 was less rewarding than Phase 2 had been, the change was gradual and did not reach the low performance level of Group S in Phase 1. While the continued eating and running in Phase 3 for all groups could be attributed to continued elicitation of these responses by cues conditioned to them in acquisition (Capaldi \& Myers, 1978), it is possible that, in addition, the pellets and/or alley had become reinforcing as a result of high-deprivation training, and responding occurred because of the continued presence of reinforcement. But, regardless of interpretation, the results of Experiment 1 indicate an empirical difference between satiation and extinction-prior experience with satiation did not increase subsequent resistance to satiation, while prior experience with nonreward clearly does increase subsequent resistance to extinction (Capaldi \& Haggbloom, 1974; Robbins, Chait, \& Weinstock, 1968; Spear, Hill, \& O'Sullivan, 1965; Spear \& Spitzner, 1967).

\section{EXPERIMENT 2}

The purpose of Experiment 2 was to determine if satiation produces a learned reduction in responding, as does extinction, suggesting that satiation is similar to extinction in being nonrewarding. Following deprived-food rewarded acquisition training, rats were tested while either satiated with food or in extinction under deprivation. In a third phase, these animals either continued the same type of testing as in Phase 2 or else were shifted to the opposite mode of testing. The question was whether a reduced tendency to respond in Phase 3 would be produced by satiated testing in Phase 2 and, if so, how this reduced tendency to respond compares with that produced by extinction in Phase 2 .

Obviously, there are gross procedural differences between the two conditions, which could independently produce performance differences in either test phase. For example, in each phase, extinction and satiation differed with respect to current deprivation level and the presence or absence of food in the alley. In addition, animals under both conditions experienced a shift from acquisition conditions in terms of deprivation and/or food schedule. The general question posed here is whether, despite these differences, extinction and satiation are functionally similar in being nonrewarding or aversive (e.g., Morgan, 1974), or, alternatively, that satiated-food testing is more rewarding than extinction. If the latter alternative is correct, less of a decrement in performance would be expected to result from satiation than from extinction.

\section{Method}

Subjects. The subjects were 50 male albino rats, 90 days old upon arrival from the Holtzman Company, Madison, Wisconsin.

Apparatus. The apparatus was the same as in Experiment 1.

Procedure. Three days following arrival in the lab (Day 1), rats were weighed and assigned randomly to 5 groups of 10 rats each. The experimental design was a 2 by 2 factorial, combining extinction (E) vs. satiation (S) in Phase 2 and Phase 3 , with the addition of a fifth group (Group S) that was not run in Phase 2 and was run while satiated in Phase 3 . The experiment was run by three experimenters-two ran three rats from each group, and the third ran four rats from each group. All rats were run while deprived (13 g of Wayne Lab Blox per day) in Phase 1 on a partial reward schedule of two rewards $(R)$ and two nonrewards $(N)$ each day. Phase 1 began on Day 12. Rats were handled individually for $90 \mathrm{sec}$ on Days 5-9, received two $.045-\mathrm{g}$ Noyes pellets in their home cages on Days 8 and 9 , and on Days 10 and 11 received one rewarded (two .045 -g pellets) running trial. In Phase 1 the following schedules were used: RNNR, NRNR, and NNRR, with this sequence repeated three times, and then the first sequence was given one additional time, for a total of 10 days. On R trials, rats were given two .045 -g pellets and were removed immediately after they consumed the pellets. On $\mathbf{N}$ trials, they were removed from the goalbox after $20 \mathrm{sec}$. Following Phase 1, Groups SS, SE, and S were given ad-lib food, and Groups EE and ES continued to receive $13 \mathrm{~g} /$ day. Phase 2 began 11 days later, when all satiated rats were at or above their Day 1 ad-lib weights, except two rats that stopped gaining weight at a level below their ad-lib weights. In Phase 2 ( 7 days of 4 trials/day), satiated groups were given two pellets on every trial and were removed from the goalbox immediately after they ate the pellets or after $20 \mathrm{sec}$, whichever came first. Rats given extinction were removed from the goalbox after $20 \mathrm{sec}$. Following Phase 2, Groups ES, SS, and $S$ received ad-lib food, while Groups $E E$ and SE were fed $13 \mathrm{~g} /$ day for 10 days, at which time all but three of the satiated rats had reached their Day 1 weights (these three had stopped gaining weight). Phase 3 also lasted 7 days ( 4 trials/day).

In all phases, rats were run randomly within squads of five rats, producing an intertrial interval of 8-10 min. Rats were given $60 \mathrm{sec}$ maximum to traverse each alley section before being moved to the next alley section. Each squad was fed its daily ration $15 \mathrm{~min}$ after its trials were completed.

\section{Results}

All times were converted to logs as in Experiment 1 . Analysis included trials and days as within factors, and experimenter, Phase 2 condition (extinction or satiation), and Phase 3 condition (extinction or satiation) as between factors. Groups was used as a between factor when Group $S$ was included in analyses in Phases 1 and 3 . Differences associated with experimenter were not significant, so this variable is not mentioned further. Results were essentially the same in alley sections, so only log total times are reported here.

Phase 1. In Phase 1, all groups decreased running times significantly $[F(9,243)=139.06, p<.001]$, including Group $S[F(9,306)=173.91, p<.001]$, and 
none of the dummy variables of Phases 2 and 3 or the interactions involving these variables reached significance.

Phase 2 and 3. Figure 2 shows the mean total running times for each group on the last day of Phase 1 and each day of Phases 2 and 3. As can be seen in Figure 2, in Phase 2, the extinction rats ran faster than the satiated rats, and the difference decreased over days. In contrast, in Phase 3, rats that had received extinction in Phase 2 (Groups EE and ES) were slower than all other groups. That is, extinction in Phase 2 produced a greater response decrement from Phase 2 to Phase 3 than did satiation in Phase 2. The groups receiving extinction in Phase 3 increased in running times over days, while the groups satiated in Phase 3 maintained relatively constant times. In general, then, extinction produced a greater response decrement than did satiation within Phase 3 (i.e., affecting current performance), and it was more persistent-extinction in Phase 2 produced a greater response decrement from Phase 2 to Phase 3.

Comparing groups within each phase, running times increased over days under both satiation and extinction in Phase $2[F(6,162)=3.58, p<.01]$ and in Phase $3[F(6,162)=3.03, p<.01$; with Group $S$, $F(6,204)=2.48, p<.05]$. The increase in running time over days in Phase 2 did not differ significantly between the groups given extinction and those given satiation $[F(6,162)=1.34]$. In Phase 3, however, the groups given extinction (Groups $\mathrm{EE}$ and $\mathrm{SE}$ ) increased in running times over days significantly more

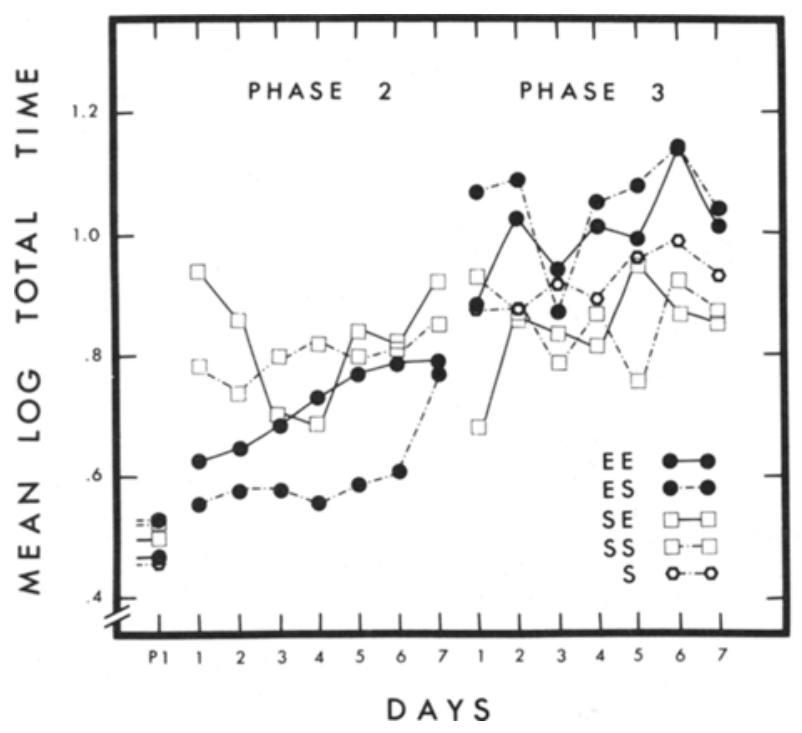

Figure 2. Mean log total times for each group on the last day of Phase 1 and for each day of Phases 2 and 3. In Phase 1, all groups were run while deprived; in Phase 2, Groups ES and EE were given extinction, Groups $S E$ and $S S$ were run while satiated, and Group $S$ was not run. In Phase 3, Groups EE and SE were given extinction, and Groups ES, SS, and S were run while satiated. than did groups given satiation $[F(6,162)=2.40$, $p<.05]$.

To examine further the effects of satiation and extinction as a function of when each was received, an analysis was conducted on Phases 2 and 3 using Phase 2 vs. Phase 3 as an additional within-subjects variable. Groups were faster in Phase 2 than in Phase $3[F(1,27)=25.28, p<.001]$. More importantly, running times increased more from Phase 2 to Phase 3 for rats given extinction in Phase 2 than for those given satiation in Phase 2. This is shown by a significant interaction between the Phase 2 condition (satiation vs. extinction) and the difference between Phase 2 and Phase 3 running times $[F(1,27)=$ $16.48, p<.001]$. Newman-Keuls tests indicated that this interaction occurred because groups given extinction in Phase 2 (Groups EE and ES) were significantly slower in Phase 3 than in Phase 2 (ps $<.01$ ), while groups given satiation in Phase 2 (Groups SS and SE) did not change significantly in running time from Phase 2 to Phase 3 (ps $>.05$ ).

It is interesting to note that, on its initial satiated test in Phase 3, Group S did not differ significantly from Groups SS and SE on their initial satiated test in Phase $2(\mathrm{~F}<1)$, despite the fact that Group S had been on ad-lib food for 21 days prior to being tested in Phase 3 and weighed $123 \%$ of its original body weight, while Groups SE and SS had been on ad-lib food 11 days prior to being tested in Phase 2 and weighed $105 \%$ of their original ad-lib weights.

Eating. Rats that were satiated ate two pellets on every trial in Phase 2 and Phase 3.

\section{Discussion}

The results of Experiment 2 support a number of conclusions about satiation and about the differences between satiation and extinction. First, it is clear that the weakening of the running response obtained in satiated testing is not critically dependent on a decline in eating. Rats ate the two pellets on every trial in satiated testing, yet running times increased over the trials of satiated testing. Views that attribute a response decrement to a decline in eating (such as that of Black \& Cox, 1973, who attribute response decrement in satiation to the gradual extinction of $r_{g}$, because of lack of eating) are strained to account for these data. In Experiment 2, eating continued even after the running response was partially extinguished (Group ES). These data support the suggestion of Capaldi and Myers (1978) that running and eating responses are learned somewhat independently. Others have also suggested that eating and running are dissociable responses (e.g., Morgan, 1974).

Second, results of Experiment 2 suggest that incomplete satiation is not an important determinant of performance under satiation. Group S, when 
tested while satiated for the first time in Phase 3, was no less resistant to satiation than Groups SE and SS had been when they were initially tested while satiated in Phase 3, despite the fact that Group S weighed $123 \%$ of their original body weights at the beginning of Phase 3 (having been on ad-lib food for 21 days at this point), while Groups SE and SS weighed $105 \%$ of their original body weights at the beginning of Phase 2. Thus, greater satiation, in terms of body weight, did not reduce responding under satiation.

Third, and most importantly, Experiment 2 showed that a greater response decrement was produced by extinction than by satiation. This was true both in current performance as a function of satiation vs. extinction and in the persistent effect of satiation or extinction on later performance. That is, performance in Phase 3 was reduced more by having had extinction in Phase 2 than by having had satiation, regardless of whether Phase 3 was extinction or satiation. These results are consistent with the hypothesis that extinction is more nonrewarding or aversive than satiation. In addition, these results suggest that this difference between satiation and extinction produces not only a performance difference, but also a difference in learning. Extinction in Phase 2 produced a greater learned response decrement (as measured in the subsequent Phase 3 test phase) than did satiation. Whether this difference reflects learned competing responses (e.g., Amsel, 1958) or greater conditioned inhibition (e.g., Konorski's "no US," as well as antidrive in extinction vs. only antidrive in satiation) or some other difference in learning, it does not seem likely that one can interpret extinction and satiation as affecting performance through a single, common underlying mechanism. As shown here, in both experiments, there are important empirical differences between the effects of the procedures of satiation and extinction, differences that suggest that satiation is less nonrewarding or aversive than extinction. More recent results that we have obtained suggest that eating under satiation following deprived acquisition may even be rewarding (see Capaldi, Davidson, \& Myers, in press).

Thus, while it seems clear that there are similarities between effects of satiation and extinction that can be interpreted as reflecting the reduced reward or reduced appetitive motivation common to both procedures (Capaldi \& Myers, 1978; Morgan, 1974), it seems equally clear that there are also differences between satiation and extinction. These differences seem most likely attributable to additional processes occurring in extinction, which do not occur in satiation (e.g., frustration, Amsel, 1958; arousal of an aversive motivational system that inhibits appetitive motivation, Dickinson \& Dearing, 1979; or conditioned inhibition, Konorski, 1967). These processes produce a greater response decrement in extinction than in satiation.

\section{REFERENCES}

Allport, G. W. Personality. New York: Holt, 1937.

Amse L, A. The role of frustrative nonreward in noncontinuous reward situations. Psychological Bulletin, 1958, 55, 102-119.

Black, R. W., \& Cox, M. Extinction of an instrumental running response in rats in the absence of frustration and nonreinforcement. Psychological Record, 1973, 23, 101-109.

Bolles, R. C. Theory of motivation (2nd ed.). New York: Harper \& Row, 1975.

Capaldi, E. D., Davidson, T. L., \& Myers, D. E. Resistance to satiation: Reinforcing effects of food and eating under satiation. Learning and Motivation, in press.

CAPAldi, E. D., \& MYERs, D. E. Resistance to satiation of consummatory and instrumental performance. Learning and Motivation, 1978, 9, 179-201.

Capaldi, E. D., \& Myers, D. E. Resistance to satiation as a function of three different satiation procedures. Bulletin of the Psychonomic Society, 1979, 14, 53-56.

Capaldi, E. J., \& Haggbloom, S. J. Influence of reward magnitude on the initial nonreward effect. Bulletin of the Psychonomic Society, 1974, 3, 219-221.

Cox, W. M. Eight drive-reward combinations: A test of incentive motivational theory. Bulletin of the Psychonomic Society, 1976, $7,121-124$.

Cravens, R. W., \& Renner, K. E. Conditioned appetitive drive states: Empirical evidence and theoretical status. Psychological Bulletin, 1970, 73, 212-220.

DaLy, H. B. Reinforcing properties of escape from frustration aroused in various learning situations. In G. H. Bower (Ed.), The psychology of learning and motivation (Vol. 8). New York: Academic Press, 1974.

Dickinson, A., \& Dearing, M. F. Appetitive-aversive interactions and inhibitory processes. In A. Dickinson \& R. A. Boakes (Eds.), Mechanisms of learning and motivation: $A$ memorial volume to Jerzy Konorski. Hillsdale, N.J: Erlbaum, 1979.

Hul,L, C. L. Principles of behavior. New York: Appleton-CenturyCrofts, 1943.

Konorski, J. Integrative activity of the brain: An interdisciplinary approach. Chicago: University of Chicago Press, 1967.

Mandle R, G. The interruption of behavior. In D. Levine (Ed.), Nebraska Symposium on Motivation. Lincoln: University of Nebraska Press, 1964.

Morgan, M. J. Resistance to satiation. Animal Behaviour, 1974, 22, 449-466.

Morris, R. G. M., Einon, D. F., \& Morgan, M. J. Persistent behavior in extinction after partial deprivation in training. Quarterly Journal of Experimental Psychology, 1976, 28, 633642.

Robbins, D., Chait, H., \& Weinstock, S. Effects of nonreinforcement on running behavior during acquisition, extinction, and reacquisition. Journal of Comparative and Physiological Psychology, 1968, 66, 699-706.

Seward, J. P., Shea, R. A., \& Elkind, D. Evidence for the interaction of drive and reward. American Journal of Psychology, $1958,71,404-407$

Spear, N. E., Hill, W. F., \& O'Sullivan, D. J. Acquisition and extinction after initial trials without reward. Journal of Experimental Psychology, 1965, 69, 24-29.

Spear, N. E., \& Spitzner, J. H. Effect of initial nonrewarded trials: Factors responsible for increased resistance to extinction. Journal of Experimental Psychology, 1967, 74, 525-537.

WEBв, W. B, Responses in the absence of the acquisition motive. Psychological Review, 1952, 59, 54-61.

(Received for publication April 20, 1980; revision accepted October $8,1980$. ) 